\title{
Questions prioritaires en matière de recherche sur le cancer d'origine professionnelle : points de vue des intervenants en Ontario
}

\author{
K. Hohenadel, M. Sc. (1); E. Pichora, M. Sc. (2); L. Marrett, Ph. D. (1,2,3); D. Bukvic, M.P.H. (1); J. Brown, M.S.O.D. (1); \\ S. A. Harris, Ph. D. (1,2,3); P. A. Demers, Ph. D. (1,2,3); A. Blair, Ph. D. (1)
}

Cet article a fait l'objet d'une évaluation par les pairs.

\section{Résumé}

Introduction : En milieu de travail, les travailleurs peuvent être exposés à des agents cancérogènes connus ou présumés, dont bon nombre n’ont pas fait l'objet d'une évaluation complète. La recherche sur le cancer d'origine professionnelle a connu une baisse au cours des dernières décennies, et ce, même si les besoins demeurent constants. Le Centre de recherche sur le cancer professionnel (CRCP) a été créé en vue de contrer cette tendance à la baisse en Ontario. Le CRCP a mené une enquête auprès de l'ensemble des intervenants pour connaître les questions prioritaires en matière de recherche sur le cancer d'origine professionnelle.

Méthodologie : Dans le cadre de l'enquête, le CRCP a reçu 177 réponses provenant d'intervenants du milieu universitaire, du milieu de la santé et de l'industrie, d'intervenants affiliés à des syndicats et de responsables des politiques. Les réponses ont été analysées en fonction de l'exposition en milieu de travail, des emplois à risque et du siège de cancer, et elles ont été stratifiées selon le poste occupé.

Résultats : Les questions prioritaires soulevées comprennent diverses catégories d'exposition en milieu de travail, notamment les catégories "produits chimiques ", "particules et fibres inhalables » (p. ex. amiante), " rayonnements » (p. ex. champs électromagnétiques), "pesticides » et " travail par quarts », ainsi que divers types d'emplois, en particulier les mineurs, les travailleurs de la construction et les travailleurs de la santé. Un financement inadéquat et des données insuffisantes sur l'exposition ont été définis comme les principaux obstacles à la recherche sur le cancer d'origine professionnelle.

Discussion : Les résultats de cette enquête font ressortir le besoin criant de recherche sur le cancer d'origine professionnelle en Ontario et ailleurs. Ces données seront très utiles pour l'élaboration du programme de recherche du CRCP.

Mots clés : cancer, emploi, milieu de travail, consultation, Ontario

\section{Introduction}

Le Centre international de recherche sur le cancer (CIRC) a classé approximativement 60 agents présents en milieu de travail comme des cancérogènes connus ou pré- sumés pour l'être humain et a recensé plus de 100 agents comme des cancérogènes possibles en milieu de travail1. Selon les estimations initiales du projet CAREX Canada ${ }^{2}$
(CARcinogen Exposure, c'est-à-dire exposition aux agents cancérogènes), des centaines de milliers de travailleurs de l'Ontario sont actuellement exposés à des agents cancérogènes connus ou présumés. Comme cette population continue de croître, un plus grand nombre de travailleurs pourrait être exposé à des agents cancérogènes. Bien que le nombre précis de cancers d'origine professionnelle au Canada ne soit pas connu, on estime qu'entre $4 \%$ et $10 \%$ des décès dus au cancer dans les pays développés pourraient être attribuables à une exposition professionnelle évitable ${ }^{3}$.

Malgré les progrès remarquables dans le recensement des agents cancérogènes pour l'être humain, grâce à des d'études sur l'exposition professionnelle ${ }^{1}$, les efforts visant à recenser et à caractériser les agents cancérogènes en milieu de travail ont été moins importants au cours des dernières décennies ${ }^{4,5,6}$. De nouveaux projets de recherche sont nécessaires pour découvrir d'autres agents cancérogènes et mieux caractériser les agents cancérogènes présumés, déterminer les milieux de travail touchés, estimer le nombre de travailleurs exposés à ces agents et les types de cancers causés et, enfin, mettre en place et évaluer des me-sures de prévention ${ }^{3,6}$.

Le Centre de recherche sur le cancer professionnel (CRCP) a été créé début 2009 pour répondre à ces besoins en Ontario. Le rôle du CRCP consiste à recenser les agents cancérogènes et à prévenir, voire à éliminer, l'exposition à ceux-ci en milieu de travail en menant des activités de surveil- 
lance, en effectuant des travaux de recherche sur l'étiologie et sur les interventions, ainsi qu'en favorisant le transfert des connaissances. Le financement du CRCP est assuré par Action Cancer Ontario (ACO), l'organisme ontarien responsable de l'amélioration des services de cancérologie, par la division ontarienne de la Société canadienne du cancer (SCC), un organisme à but non lucratif, et par la Commission de la sécurité professionnelle et de l'assurance contre les accidents du travail (CSPAAT) de l'Ontario. Le CRCP a été créé en collaboration avec le Syndicat international des travailleurs unis de la métallurgie. La CSPAAT accorde également du financement à plusieurs autres centres de recherche qui s'intéressent à d'autres domaines de la santé au travail, notamment l'Institut de recherche sur le travail et la santé, le Centre d'expertise en recherche sur les maladies professionnelles et le Centre d'expertise en recherche pour la prévention des troubles musculosquelettiques.

Une communauté vaste et diversifiée d'intervenants appuie également le CRCP : universitaires et chercheurs, syndicats et travailleurs, employeurs, professionnels de la santé, décideurs/défenseurs de politiques, spécialistes en santé et en sécurité, hygiénistes du travail et membres du public qui s’intéressent à la santé au travail en général.

Les organismes de recherche utilisent traditionnellement diverses stratégies pour déterminer les priorités en matière de recherche sur la santé en milieu de travail, dont la revue des documents publiés, la consultation de spécialistes sur les normes et, très souvent, les systèmes de cotation utilisés par les spécialistes pour arriver à un consensus sur des domaines prioritaires $^{7,8,9,10}$. Comme le CRCP a été créé en partie pour répondre aux besoins de l'ensemble des intervenants, nous considérons qu'il est important qu'il participe à l'élaboration d'une stratégie de recherche. En conséquence, l'un des premiers projets du CRCP a été de consulter les intervenants qui s'intéressent à la prévention du cancer d'origine professionnelle en vue de comprendre leurs différents points de vue concernant les priorités en matière de recherche sur le cancer d'origine professionnelle en Ontario, et d'utiliser ces données, tout comme les lacunes connues liées à notre compré-hension du processus cancérogène, pour élaborer un programme de recherche complet pour le CRCP.

La consultation des intervenants réalisée par le CRCP s'est faite par une enquête en ligne destinée à l'ensemble des intervenants en Ontario ainsi qu'à ceux qui vivent ou travaillent à l'extérieur de la province mais sont en réseau avec ces intervenants, ainsi que par des entrevues de suivi ciblées visant un petit nombre de répondants afin d'obtenir des commentaires additionnels. Cet article porte principalement sur les résultats de l'enquête en ligne.

\section{Méthodologie}

\section{Enquête}

Le CRCP a informé de l'enquête les personnes qui s'intéressent au cancer d'origine professionnelle en Ontario. Cette enquête comprenait une série de questions ouvertes sur le point de vue des répondants concernant les questions prioritaires en matière de recherche sur le cancer d'origine professionnelle, sur les obstacles perçus concernant la recherche sur le cancer d'origine professionnelle et les solutions possibles pour les surmonter ainsi que sur les types de travaux de recherche en cours et les façons dont les intervenants aimeraient collaborer avec le CRCP. L'enquête recueillait également, au moyen de questions à choix multiples, des informations sur le lieu de résidence, le rôle professionnel et l'affiliation professionnelle des répondants.

Le protocole et le questionnaire de l'enquête ont été élaborés conjointement avec le Comité de direction et le Comité consultatif scientifique du CRCP. Le Bureau d'éthique de la recherche de l'Université de Toronto a déclaré qu'une évaluation éthique du projet n’était pas nécessaire.

\section{Mise en auvre de l'enquête}

L'enquête en ligne, élaborée au moyen de l'outil SurveyMonkey, a pu être remplie du 6 juin au 25 juillet 2009. Une copie papier était également offerte sur demande pendant la même période. L'information sur l'enquête a été transmise grâce à une liste de diffusion créée par le CRCP à l'intention des partenaires établis parmi les bailleurs de fonds et les autres collaborateurs, ainsi que des représentants du milieu universitaire, de l'industrie, de syndicats, d'organisations de travailleurs, d'établissements de soins de santé et d'organismes gouvernementaux. Pour s'assurer que la liste de diffusion comprenait des chercheurs actifs dans le domaine, le CRCP a procédé à l'analyse de la recherche sur le cancer d'origine professionnelle financée par sept organismes de financement canadiens pertinents entre 2004 et 2009. Comme le CRCP souhaitait rejoindre le plus grand nombre possible d'intervenants, les membres de la liste de diffusion à qui le courriel sur l'enquête avait été envoyé ont été encouragés à transmettre le lien à d'autres membres de leur réseau. En conséquence, il n'est pas possible de déterminer le nombre exact de personnes qui ont été invitées à participer à l'enquête.

\section{Analyse}

Sous la supervision du directeur intérimaire, deux associés de recherche ont regroupé par thème les réponses aux questions ouvertes avant de mettre en tableaux les données sur la fréquence. Nous avons regroupé les données sur le type d'exposition en fonction de la liste établie dans l'étude de Siemiatycki et collab. ${ }^{1}$, du poste occupé comme dans la Classification type des professions de $2000^{11}$ et du siège de cancer. Nous avons calculé les fréquences à l'aide de la version $9.2 \mathrm{du}$ logiciel statistique SAS (SAS Institute Inc.).

Nous avons également stratifié les réponses selon le rôle professionnel des répondants pour avoir un point de comparaison. Pour cela, nous avons classé les répondants ayant choisi plus d'un rôle dans le groupe qui figurait en premier lieu selon un ordre préétabli, soit travailleur, chercheur/scientifique, spécialiste en santé et en sécurité, hygiéniste industriel, professionnel de la santé et citoyen intéressé. Nous n'avons pas inclus dans les analyses stratifiées les répondants qui n'avaient pas indiqué leur rôle professionnel ( $\mathrm{n}=17$ ) ou qui ne faisaient pas partie de l'un de ces groupes $(n=20)$.

\footnotetext{
* Le questionnaire de l'enquête est disponible sur demande.
} 


\section{Résultats}

Sur les 192 réponses à l'enquête reçues, nous en avons exclu 15 pour lesquelles il manquait un grand nombre de variables, de sorte que 177 questionnaires ont été analysés. La majorité des répondants (52 \%) avaient reçu un courriel du personnel du CRCP renfermant un lien vers le questionnaire de l'enquête. Un autre groupe (24\%) avait été informé de la tenue de l'enquête par un collègue. Un répondant avait trouvé par lui-même le lien permettant d'accéder à l'enquête dans le site Web du CRCP. Dans le cas des autres répondants (24\%), le lien a été envoyé par un autre groupe d'intervenants, une autre organisation ou une autre industrie.

\section{Caractéristiques des répondants}

La plupart des répondants (72\%) vivaient en Ontario, mais près de $10 \%$ vivaient dans une autre province (ColombieBritannique, Alberta, Manitoba, NouvelleÉcosse, Québec, Nouveau-Brunswick ou Terre-Neuve-et-Labrador) et près de $5 \%$ vivaient dans un autre pays. Bien que les chercheurs et les scientifiques des établissements universitaires ainsi que les autres spécialistes en santé et en sécurité aient constitué le groupe le plus important de répondants, on retrouvait parmi ceux-ci des affiliations et des rôles professionnels variés (tableau 1).

\section{Questions prioritaires en matière de recherche sur le cancer d'origine professionnelle}

Exposition. Nous avons recensé près de 100 types notables d'exposition à divers niveaux de spécificité (voir tableau 2), allant du mélange de cancérogènes bien établis (comme l'amiante ou le benzène) à des questions émergentes comme le travail par quarts et la nanotechnologie. Plusieurs types d'exposition mentionnés par les répondants n'ont pas fait l'objet d'une évaluation approfondie en vue d'établir un lien avec le cancer et, dans certains cas, il est peu probable qu'il existe un lien de cause à effet.

Les réponses stratifiées selon le rôle professionnel des répondants ont révélé une préoccupation commune pour plusieurs grands types d'exposition. Tous les groupes ont mentionné les carburants et les gaz
TABLEAU 1

Caractéristiques des intervenants qui ont répondu au questionnaire du CRCP

$(\mathbf{N}=177)$

\begin{tabular}{|c|c|c|c|}
\hline & \multirow{4}{*}{$\begin{array}{l}\text { particules, mais ils les ont toutefois men- } \\
\text { tionnées fréquemment; enfin, dans tous les } \\
\text { groupes, exception faite des travailleurs et } \\
\text { des citoyens intéressés, le travail par quarts } \\
\text { a été mentionné souvent. }\end{array}$} \\
\hline Caractéristique & $\begin{array}{c}\text { Nombre, } \\
n\end{array}$ & $\begin{array}{c}\text { Pourcentage de } \\
\text { répondants, } \\
\%\end{array}$ & \\
\hline \multicolumn{3}{|l|}{ Localisation $^{\mathrm{a}}$} & \\
\hline Canada & & & \\
\hline
\end{tabular}

\begin{tabular}{lrr} 
Ontario & 127 & 71,8 \\
Colombie- & 4 & 2,3 \\
Britannique & & \\
Alberta & 3 & 1,7 \\
Manitoba & 3 & 1,7 \\
Nouvelle-Écosse & 3 & 1,7 \\
Québec & 2 & 1,1 \\
Nouveau- & 1 & 0,6 \\
Brunswick & & \\
Terre-Neuve-et- & 1 & 0,6 \\
$\quad$ Labrador & & \\
International & 8 & 4,5 \\
Non précisé & 25 & 14,1 \\
\hline Sition
\end{tabular}

\begin{tabular}{l}
$\begin{array}{l}\text { Situation } \\
\text { professionnelle }^{\mathrm{b}}\end{array}$ \\
\hline
\end{tabular}

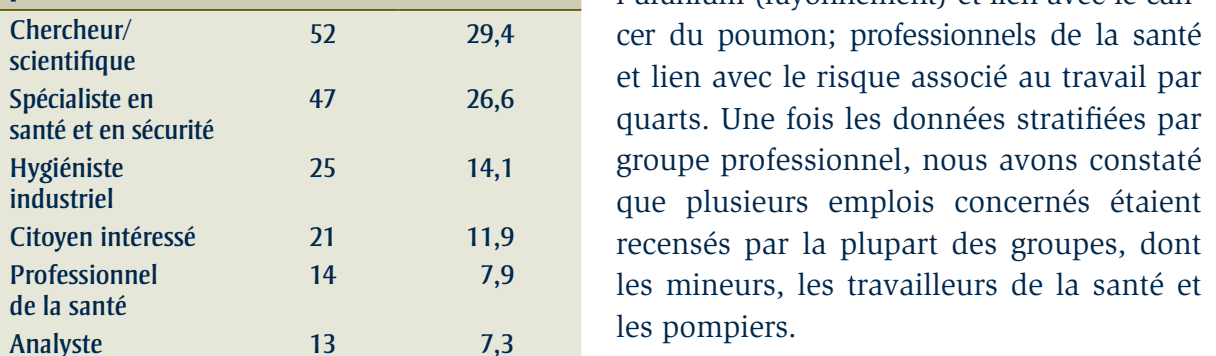

\section{des politiques}

Spécialiste de

l'application des

6,8

connaissances

$\begin{array}{lrr}\text { Travailleur } & 12 & 6,8 \\ \text { Employeur } & 5 & 2,8\end{array}$

\begin{tabular}{|c|c|c|}
\hline $\begin{array}{l}\text { Affiliation } \\
\text { professionnelle }\end{array}$ & & \\
\hline $\begin{array}{l}\text { Établissement } \\
\text { universitaire }\end{array}$ & 45 & 25,4 \\
\hline Gouvernement & 24 & 13,6 \\
\hline Syndicat & 23 & 13,0 \\
\hline $\begin{array}{l}\text { Organisation non } \\
\text { gouvernementale }\end{array}$ & 21 & 11,9 \\
\hline Industrie & 18 & 10,2 \\
\hline $\begin{array}{l}\text { Organisation } \\
\text { responsable de } \\
\text { la santé et de } \\
\text { la sécurité }\end{array}$ & 15 & 8,5 \\
\hline $\begin{array}{l}\text { Établissement } \\
\text { de santé }\end{array}$ & 14 & 7,9 \\
\hline Aucune affiliation & 6 & 3,4 \\
\hline
\end{tabular}

a Les chiffres étant arrondis, il se peut que la somme des pourcentages ne soit pas égale à $100 \%$.

Les répondants pouvaient sélectionner plus d'une situation professionnelle et plus d'une affiliation professionnelle.

d'échappement, l'air et l'eau contaminés ainsi que l'amiante; tous les groupes, sauf les professionnels de la santé, ont mentionné les produits chimiques (en général); santé et en sécurité ont mentionné les nano-

Emplois. De nombreux répondants ont suggéré certains emplois comme sujet de recherche en lien avec le risque de cancer : ils en ont énuméré 45 , aussi bien des emplois généraux que des emplois spécifiques (voir tableau 2). Les répondants ont également mentionné plusieurs emplois associés à des expositions ou à des cancers spécifiques : paysagistes, travailleurs agricoles et agriculteurs et lien avec l'exposition à des pesticides; mineurs exposés à la silice ou à l'uranium (rayonnement) et lien avec le cancer du poumon; professionnels de la santé et lien avec le risque associé au travail par quarts. Une fois les données stratifiées par groupe professionnel, nous avons constaté que plusieurs emplois concernés étaient recensés par la plupart des groupes, dont Cancers. Une faible proportion des répondants a mentionné des cancers précis comme priorités en matière de recherche sur les cancers d'origine professionnelle, en tout 27 types de cancers à divers degrés de spécificité ont été nommés (tableau 2). Le cancer du sein a été le plus fréquemment cité, et a été cité par les répondants de tous les groupes professionnels. Les différences entre les groupes portaient sinon sur le choix des cancers définis comme prioritaires. Les citoyens intéressés ont mentionné plusieurs cancers précis non énumérés par d'autres groupes, dont le lymphome cutané, le myélome multiple et l'adénocarcinome pulmonaire. Les chercheurs et les spécialistes en santé et en sécurité ont été les seuls groupes à mentionner le mésothéliome, un cancer fortement lié à l'exposition à l'amiante en milieu de travail; le cancer du sein et le cancer de la prostate ont été énumérés fréquemment en lien avec le travail par quarts, en particulier chez les professionnels de la santé. seuls les chercheurs et les spécialistes en 
TABLEAU 2

Questions prioritaires en matière de recherche mentionnées par les répondants, selon la catégorie d'exposition, le groupe professionnel et le siège du cancer

\begin{tabular}{|c|c|c|}
\hline Exposition & $\underset{n}{\text { Nombre }}$ & \\
\hline Catégorie d'exposition & & Principales expositions mentionnées ${ }^{b}$ \\
\hline Produits chimiques & 30 & - \\
\hline Particules et fibres inhalables & 27 & Amiante, fibre de verre, silice \\
\hline Rayonnement & 24 & $\begin{array}{l}\text { Champs électromagnétiques, rayonnement } \\
\text { nucléaire, téléphone cellulaire, ordinateur, soleil }\end{array}$ \\
\hline Travail par quarts & 16 & - \\
\hline Pesticides & 15 & - \\
\hline Nanomatériaux & 14 & - \\
\hline Émissions & 14 & Diesel, essence \\
\hline Métaux et composés métalliques & 13 & - \\
\hline Milieu de travail & 12 & Air intérieur, fumé secondaire du tabac \\
\hline Solvants & 9 & Solvants, benzène \\
\hline $\begin{array}{l}\text { Bois, combustibles fossiles } \\
\text { et produits pétroliers }\end{array}$ & 7 & - \\
\hline Produits pharmaceutiques & 4 & Médicaments antinéoplastiques \\
\hline Plastique et caoutchouc & 4 & - \\
\hline Expositions liées à la préparation d'aliments & 2 & - \\
\hline Principaux groupes d'emploi & & Principaux emplois/domaines mentionnés ${ }^{b}$ \\
\hline Construction et extraction & 25 & Mineur, travailleur de la construction, peintre \\
\hline Domaine de la santé & 20 & Travailleur de la santé, soignant \\
\hline Production & 14 & Soudeur, technicien nucléaire \\
\hline Services de protection & 10 & Pompier \\
\hline $\begin{array}{l}\text { Industrie agricole, industrie forestière, } \\
\text { industrie de la pêche }\end{array}$ & 9 & Agriculteur, travailleur agricole \\
\hline Installation, entretien, réparation & 5 & Mécanicien \\
\hline $\begin{array}{l}\text { Entretien de bâtiments et } \\
\text { entretien paysager }\end{array}$ & 4 & Paysagiste \\
\hline Transport & 3 & - \\
\hline Informatique et mathématiques & 2 & - \\
\hline Préparation et service des aliments & 2 & Employé de restaurant \\
\hline Affaires et finances & 1 & - \\
\hline Services de soins personnels & 1 & - \\
\hline Siège de cancer & & Sous-types \\
\hline Sein & 17 & - \\
\hline Appareil respiratoire & 14 & $\begin{array}{l}\text { Poumons, larynx, adénocarcinomes } \\
\text { du poumon, nez }\end{array}$ \\
\hline Système hématopoïétique & 10 & $\begin{array}{l}\text { LNH, lymphome, LMA, lymphome cutané, } \\
\text { leucémie, myélome multiple }\end{array}$ \\
\hline Appareil génital & 9 & Prostate, ovaires, testicules \\
\hline Appareil digestif & 5 & Côlon, œesophage, foie, pancréas \\
\hline Cerveau & 4 & - \\
\hline Peau & 3 & - \\
\hline Mésothéliome & 2 & - \\
\hline Appareil urinaire & 2 & Vessie \\
\hline Cancer chez l'enfant & 2 & Cancers infantiles, neuroblastome \\
\hline Autre & 2 & Sarcome, thyroïde \\
\hline
\end{tabular}

${ }^{a}$ Nombre de répondants ayant mentionné chaque exposition, emploi ou type de cancer.

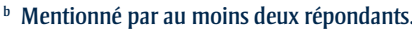

Autres questions prioritaires en matière de recherche. De nombreux répondants (32\%) estiment que le CRCP devrait élaborer des ressources spécifiques à l'intention des chercheurs et des autres intervenants, notamment des bases de données sur l'exposition, des registres de maladies et des cartes créées au moyen d'un système d'information géographique (SIG). D'autres répondants ont recommandé d'axer les efforts sur la prévention (28\%) ou d'utiliser des méthodes précises ou certains modèles d'études (25\%), par exemple des études de cohortes à long terme, des études reposant sur des méthodes mixtes et la biosurveillance. Plusieurs répondants (12\%) ont mentionné la nécessité d'évaluer l'interaction entre deux expositions ou plus, entre les expositions et les gènes ou entre les expositions et les facteurs liés au style de vie comme l'alimentation, le tabagisme et les infections virales. Tous les groupes ont cité la mise en œuvre ou l'amélioration des efforts de prévention parmi les questions prioritaires les plus importantes, et tous les groupes sauf les professionnels de la santé ont fait état de projets de recherche spécifiques qui devraient être prioritaires.

\section{Obstacles à la recherche sur le cancer d'origine professionnelle en Ontario}

Les intervenants ont donné leur avis sur certains obstacles courants à la recherche sur le cancer d'origine professionnelle en Ontario et ont proposé des solutions pour les surmonter. Tous les groupes et les intervenants ont mentionné le manque de financement comme principal obstacle à la réalisation de la recherche sur le cancer d'origine professionnelle. Un autre thème récurrent a été le peu de données dont on dispose sur les expositions et les effets, ainsi que les difficultés associées à l'application dans le milieu de travail des résultats de la recherche sur le cancer d'origine professionnelle pour en réduire le risque. Parmi les autres obstacles cités, mentionnons le manque d'information sur les questions liées au cancer d'origine professionnelle, la résistance de l'employeur ou de l'industrie, le fait qu'il est difficile de distinguer les relations entre l'exposition et les effets, le faible intérêt du public et des politiciens face à la question, le manque de collaboration et la petite taille des populations dans les études.

La solution la plus souvent citée pour surmonter ces obstacles a été de favoriser la collaboration entre les groupes des différentes régions géographiques et disciplines, c'est-à-dire les chercheurs avec les employeurs et les employés, les chercheurs avec les décideurs de politiques et les syndicats et enfin les intervenants avec les chercheurs. Les autres solutions les plus 
couramment suggérées ont été la sensibilisation aux agents cancérogènes d'origine professionnelle, l'accroissement de la formation et de l'éducation ainsi que le renforcement des politiques et de la réglementation. Les chercheurs et les professionnels de la santé ont mentionné la collaboration entre les différents groupes comme étant la solution essentielle, alors que les spécialistes en santé et en sécurité et les citoyens intéressés ont indiqué que le plus important était la sensibilisation et l'éducation. La nécessité pour le gouvernement d'accorder la priorité à la question figurait en tête de liste pour les travailleurs, solution qui n’a été proposée par aucun autre groupe.

\section{Discussion}

La consultation des intervenants du CRCP a permis de générer une longue liste d'expositions, d'emplois, de cancers et d'autres questions que les intervenants considèrent comme prioritaires en matière de recherche sur le cancer d'origine professionnelle. Les priorités les plus importantes mentionnées par les répondants, à savoir les produits chimiques ainsi que les particules et les fibres inhalables, n'ont rien d'inattendu, car ces agents sont présents dans de nombreux lieux de travail. Parmi les autres priorités recensées, on trouve à la fois des agents cancérogènes bien établis (l'amiante et les rayonnements), des agents cancérogènes présumés mais non reconnus (les pesticides et certains solvants), des facteurs qui suscitent actuellement l'intérêt (le travail par quarts) et les expositions émergentes dont les effets sont toujours en grande partie inconnus (les nanomatériaux).

Bon nombre des priorités en matière d'exposition mentionnées par les intervenants du CRCP rejoignent les priorités concernant la recherche établies par le programme National Occupational Research Agenda (NORA) des États-Unis, par exemple la nécessité de mieux caractériser les agents que l'on soupçonne d'être cancérogènes (p. ex. les produits chimiques), de recenser les agents cancérogènes émergents (p. ex. les nanomatériaux) et de continuer d'assurer la surveillance des agents cancérogènes d'origine professionnelle connus (p. ex. l'amiante) ${ }^{3}$. En outre, CAREX Canada a déterminé que de nombreuses expositions précises mentionnées par les intervenants du CRCP comptent parmi celles qui affichent la prévalence la plus élevée en Ontario, soit le travail par quarts (entre 745000 et 1051000 travailleurs exposés, selon la définition utilisée), les émissions de diesel (275 000 travailleurs exposés), le benzène (112 000 travailleurs exposés) et l'amiante (52 000 travailleurs exposés) ${ }^{13}$.

Lorsque nous avons stratifié les priorités selon le rôle professionnel, nous avons constaté que les répondants se présentant comme des travailleurs ou des citoyens intéressés avaient tendance à mentionner des agents cancérogènes bien établis, tandis que les chercheurs, les hygiénistes industriels et les spécialistes en santé et en sécurité mentionnaient ces mêmes agents cancérogènes sur le même plan que d'autres dangers émergents. Ces divergences pourraient s'expliquer par des différences liées à l'accès à l'information, mais pourraient aussi être attribuables à la taille de l'échantillon ou à la participation inégale des différents groupes.

Les postes les plus souvent cités dans les priorités en matière de recherche sur le cancer comprenaient à la fois des emplois présentant des liens bien établis avec le cancer, comme ceux des domaines de la construction et de l'extraction, et des emplois dans les industries pour lesquelles un risque a été établi plus récemment, comme le travail dans le secteur de la santé. Dans la catégorie de la construction et de l'extraction, les répondants ont mentionné notamment le travail dans l'industrie minière, le domaine de la construction ou le travail de peintre, dont certains types d'emploi ont été classés comme associés au Groupe 1 par le CIRC (agents cancérogènes pour l'homme) ${ }^{12}$. Le secteur de la santé, qui n’a jamais été considéré comme un secteur présentant un risque élevé de cancer, s'est classé au second rang des priorités en matière de recherche sur le cancer d'origine professionnelle. Cet ordre de priorité est particulièrement intéressant compte tenu du fait que l'on se préoccupe davantage des questions concernant le travail par quarts et l'exposition à des agents antinéoplasiques au sein de l'industrie de la santé, questions qui ont toutes deux été mentionnées par les répondants comme des préoccupations. Les autres emplois cités se rattachaient à des activités économiques variées : industrie de la production (soudeurs et travailleurs du secteur nucléaire), agriculture, pêche, industrie forestière, services de protection (pompiers), industrie agro-alimentaire, etc.

Les cancers les plus fréquemment mentionnés, soit le cancer du sein et les cancers respiratoires, sont également les plus courants dans la population générale. Alors qu'un lien étroit a été établi entre le cancer du poumon et de nombreuses expositions professionnelles, les liens entre les nombreuses expositions en milieu de travail et le cancer du sein sont insuffisamment documentés ${ }^{1}$. On se préoccupe pourtant de plus en plus du lien entre le travail par quarts et le cancer du sein ${ }^{14}$. Des chercheurs respectés reconnaissent que le cancer d'origine professionnelle chez la femme devrait faire l'objet d'un examen plus approfondi ${ }^{15}$.

D’autres questions définies comme prioritaires en matière de recherche sur le cancer d'origine professionnelle montrent que les répondants souhaitent voir les résultats de recherche utilisés pour améliorer les conditions de travail. Il ressort également de l'étude qu'il faut encourager et faciliter la recherche dans ce domaine par la création de bases de données et de registres. Les obstacles et les solutions mentionnés par les répondants mettent en évidence l'importance de la collaboration, de la sensibilisation et de l'utilisation de méthodes novatrices dans le cas de petites populations et de faibles expositions.

Les résultats de cette consultation seront fort utiles pour le CRCP lorsqu'il élaborera son programme de recherche, en particulier si l'on tient compte du grand nombre de bailleurs de fonds, de partenaires et d'intervenants qu'il compte. Les résultats ont déjà été utilisés pour déterminer les catégories d'exposition et les emplois prioritaires pour l'élaboration de projets et la planification d'événements. À titre d'exemple, en avril 2010, le CRCP a collaboré avec l'Institut de recherche sur le travail et la santé pour présenter un colloque sur les effets 
sur la santé du travail par quarts qui a permis de réunir des experts internationaux et qui traitait des preuves scientifiques des effets sur la santé du travail de nuit et du travail par quarts.

Les résultats de cette consultation mettent aussi en évidence les difficultés liées à l'élaboration d'un programme de recherche dans un domaine où la demande d'information est grande, mais où les communautés d'intérêts sont très variées dans les différents groupes d'intervenants. Ils font ressortir la nécessité de mener davantage de recherches sur le cancer d'origine professionnelle en Ontario comme à l'échelle nationale et internationale, d'évaluer le risque de cancer lié à l'exposition à un grand nombre d'agents cancérogènes en milieu de travail qui préoccupent les travailleurs et les groupes responsables de la santé au travail et enfin de passer de la recherche à l'action plus rapidement.

\section{Remerciements}

Les auteurs souhaitent remercier le Comité directeur et le Comité consultatif scientifique du CRCP pour leurs conseils et leur soutien, les personnes qui ont participé à la diffusion du questionnaire, de même que Sandrene ChinCheong, qui a compilé les résultats initiaux dans la base de données sur les intervenants, et Yen Borrego, qui a assuré le soutien administratif.

\section{Références}

1. Siemiatycki J, Richardson L, Straif K, Latreille B, Lakhani R, Campbell S et collab. Listing occupational carcinogens. Environ Health Perspect. 2004;112(15):1447-59.

2. CAREX Canada. Base de données sur les agents cancérogènes [Internet]. http://www .carexcanada.ca/fr/carcinogen_profiles_and _estimates [consultation le 9 janvier 2011].

3. Ward EM, Schulte PA, Bayard S, et al; National Occupational Research Agenda Team. Priorities for development of research methods in occupational cancer. Environ Health Perspect. 2003;111(1):1-12.
4. Blair A, Marret L, Beane Freeman L. Occupational cancer in developed countries. Environ Health. 2011;10 Suppl 1:S9.

5. Vineis P, Cantor K, Gonzales C, Lynge E, Vallyathan V. Occupational cancer in developed and developing countries. Int J Cancer. 1995;62:655-60.

6. Siemiatycki J. Future etiologic research in occupational cancer. Environ Health Perspect. 1995;103(S8):209-15.

7. Choi BC, Eijkemans GJ, Tennassee LM. Prioritization of occupational sentinel health events for workplace health and hazard surveillance: the Pan American health organization experience. J Occup Environ Med. 2001;43(2)147-57.

8. Harrington JM. Research priorities in occupational medicine: a survey of UK medical opinion by the Delphi technique. Occup Environ Med. 1994;51:289-94.

9. National Institute for Occupational Safety and Health. National Occupational Research Agenda. Atlanta (GA): DHHS (NIOSH); 1996. Publ. $n^{\circ}$ 96-115. Consultable en ligne à la page : http://www.cdc.gov/niosh /docs/96-115/default.html.

10. Iavicoli S, Marinaccio A, Vonesch N, Ursini CL, Grandi C, Palmi S. Research priorities in occupational health in Italy. Occup Environ Med. 2001;58:325-9.

11. U.S. Bureau of Labor Statistics. Division of Occupational Employment Statistics: Standard Occupational Classification [Internet]. Washington (DC): United States Department of Labor; 2000 [consulté le 2 juin 2010]. Consultable en ligne à la page : http://www.bls.gov/soc/2000/soc_majo.htm.

12. CIRC. Agents classés par les monographies du CIRC, Volumes 1-100. Lyon : Centre international de recherche sur le cancer [Internet]. Lyon (FR) : CIRC [mise à jour le 22 octobre 2010; consultation le 2 juin 2010]. PDF ( 98 Ko) téléchargeable à partir du lien : http://monographs.iarc.fr/ENG /Classification/ClassificationsAlphaOrder .pdf
13. Peters C. Occupational exposure to selected priority carcinogens in Ontario. Vancouver (BC): CAREX Canada; June, 2010.

14. Straif K, Baan R, Grosse $\mathrm{Y}$, Secretan B, El Ghissassi F, Bouvard V, et al. Carcinogenicity of shift-work, painting, and fire-fighting. Lancet Oncol. 2007;8:1065-6.

15. Zahm SH, Blair A. Occupational cancer among women: where have we been and where are we going? Am J Ind Med. 2003;44:565-75. 